\title{
Intramedullary Cysticercosis- An Uncommon Entity Even in the Presence of Intracranial Neurocysticercosis
}

\author{
Gurpreet Kaur Walia $^{1}$, Puneet Baveja ${ }^{1 *}$, Shipra Verma ${ }^{1}$, Sanjay Kumar ${ }^{2}$, Atul Jain ${ }^{2}$ and Rahul Dudhal ${ }^{3}$ \\ 'Dept. of Pathology, Armed Forces Medical College, Pune \\ ${ }^{2}$ Dept. of Neurosurgery, Command Hospital, Pune \\ ${ }^{3}$ Dept. of Radiology, Command Hospital, Pune
}

\begin{abstract}
Neurocysticercosis (NCC) is described as the central nervous system involvement by a parasitic infection caused by a nematode, Taenia solium. By far, NCC is the most common parasitic infection of central nervous system in the world. In the developing countries like India, NCC is a leading cause of epilepsy in all age groups. Here, we present a case of a 36-year-old male who was a known case of multiple NCC involving the brain having cognitive dysfunction and presented with difficulty in walking and sudden onset weakness of right lower limb. On contrast enhanced MRI of spine, an intramedullary lesion was seen at D7- D8 level, which was confirmed on histopathological examination of the excised tissue as NCC. During clinical follow-up, the patient has shown significant neurological improvement.
\end{abstract}

Keywords: Intramedullary, Cysticercosis, Histopathology.

\section{Introduction}

Neurocysticercosis (NCC) is described as the central nervous system involvement by a parasitic infection caused by a nematode, Taenia solium. ${ }^{[1,2]}$ Predominantly being intracranial disease, the spinal NCC is anatomically classified as extraspinal and intraspinal. Extraspinal NCC constitutes vertebral NCC, whereas, intraspinal NCC comprises of epidural, subdural intramedullary and arachnoid NCC.$^{[1,3]}$ Intraspinal involvement by NCC is very rare with an incidence of $1.5-3.0 \%$ with intramedullary being even rarer. ${ }^{[1]}$ Intramedullary lesions have significant morbidity due to limited size of spinal canal. Despite being located at a critical area of spine, these lesions are fairly treatable by resection.

\section{Case Report}

Our case is a 36 year old male who is a known case of multiple neurocysticercosis since 2008 and cognitive dysfunction since 2015. Patient had been on antiepileptic drugs after initial cysticidal therapy with steroids. In Mar 2020, he presented with difficulty in walking of one-week duration and sudden onset weakness of the right lower limb. On clinical examination, patient was disoriented with impaired higher motor functions and slurred speech. Motor examination of the limbs revealed power Medical Research Council (MRC) grade 1 in right lower limb and MRC grade 4 power in left lower limb. Sensory examination revealed $20 \%$ hypoesthesia below dorsal vertebra 10 (DV10) level.
Contrast enhanced Magnetic Resonance Imaging (CE MRI) of whole spine revealed $10 \times 7.7 \times 7.5 \mathrm{~mm}$ intramedullary lesion in dorsal spine at DV7 - DV8 level. This lesion was cystic in nature with a hypointense rim on T1W image (Fig.1a), hyperintense on T2W image with spinal cord edema (Fig.1b) and ring enhancement on contrast imaging (Fig.1c). CE MRI the brain revealed multiple ring lesions with a few lesions showing ring enhancement suggestive of active lesions (Fig 1d).

Patient underwent laminectomy DV7-DV8 with total excision of the intramedullary lesion (Fig. 2). The excised specimen was sent to our department for histopathological examination.

We received the excised specimen as two whitish colored fragmented tissue bits with the larger bit measuring $1 \times 0.8 \times 0.5 \mathrm{~cm}$ and smaller bit measuring $0.8 \times 0.2 \times 0.2 \mathrm{~cm}$. The entire tissue was processed. Hematoxylin and eosin stained sections from the processed tissue revealed a cystic cavity having a fibrous pseudocapsule containing the larval scolex with duct-like invaginations lined by double layered eosinophilic membrane. Cystic cavity also showed presence of hooklets with numerous calcareous bodies. One pair of suckers was noted at the periphery (Fig. 3a). Moderate amount of inflammatory infiltrate comprising predominantly of lymphocytes and plasma cells was noted in the surrounding tissue (Fig. 3b). No giant cells/ granulomas were noted. Based on above mentioned histopathological findings, a diagnosis of intramedullary neurocysticercosis was given. 
Post-operatively, patient's neurological deficit has gradually improved with power of MRC grade 4 in right lower limb with gradual improvement in neurological deficit. He is presently being continued on anti-epileptics.

\section{Discussion}

The most common parasitic central nervous system involvement, neurocysticercosis (NCC) is caused by the larval form of a nematode, Taenia solium. ${ }^{[1,2]} \mathrm{NCC}$ is endemic in various low socio-economic countries like Africa, Mexico, Latin America and Asia, including India. [4,5] NCC is considered to be the most common cause of acquired epilepsy as $80 \%$ of cases present with seizures. ${ }^{[6,7]}$ $\mathrm{NCC}$ is caused either by ingestion of food contaminated with eggs of Taenia solium or through feco-oral spread from faecal matter of carriers or infected individuals.
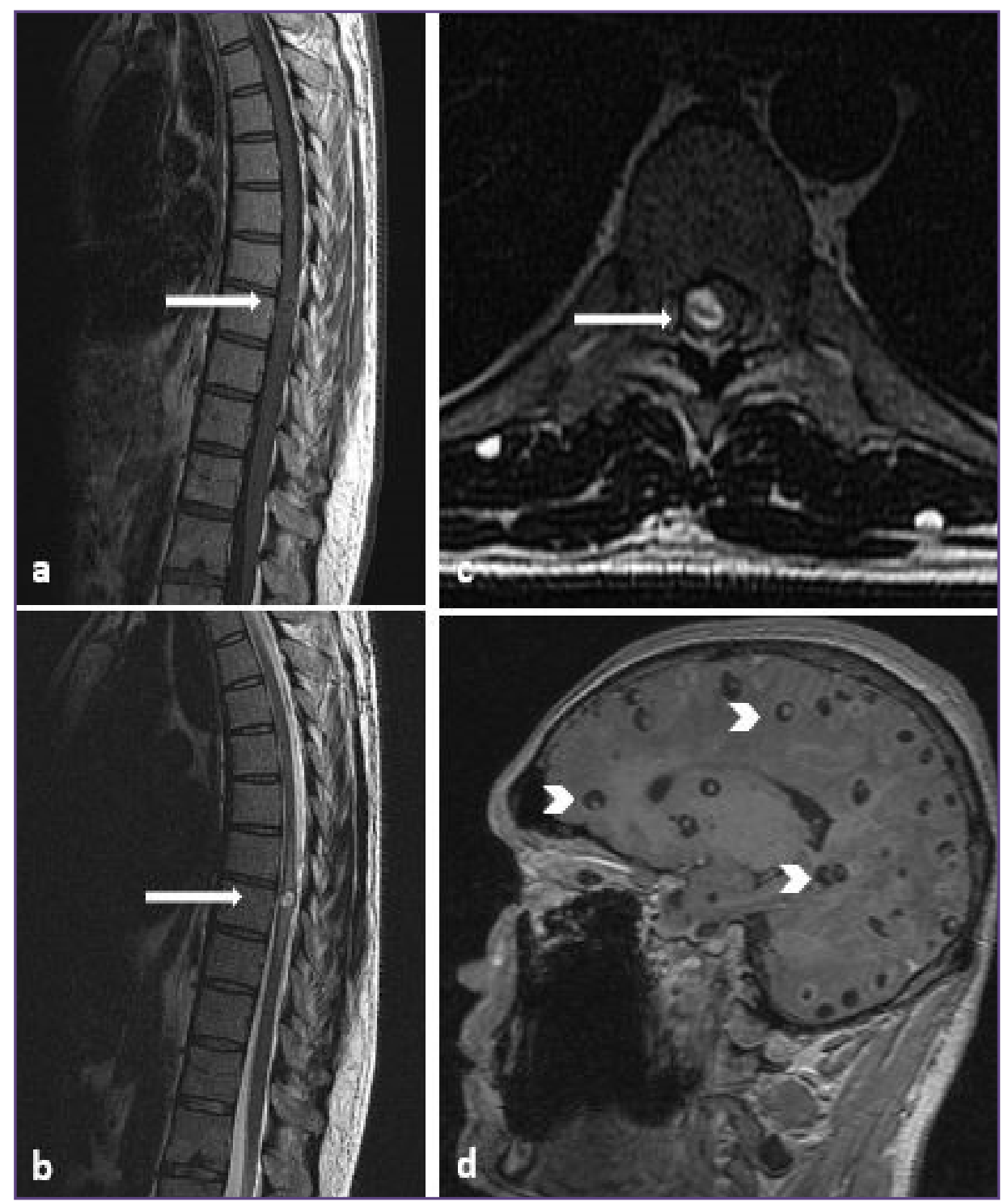

Fig. 1(a-d): MRI images of brain and spinal cord; (a-b) T1W and T2W sagittal images showing ring lesion with hypointense and hyperintense rim, respectively;(c) post contrast axial image showing contrast enhancement; (d) T1W post contrast sagittal image of brain showing multiple lesions.

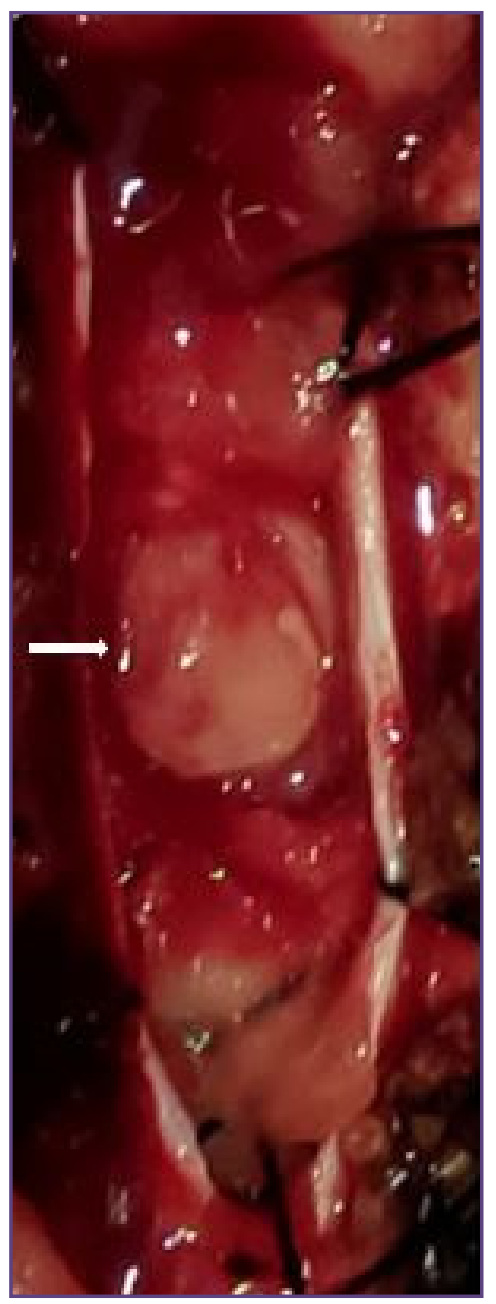

Fig. 2: Intraoperative image showing intramedullary lesion after midline myelotomy. 


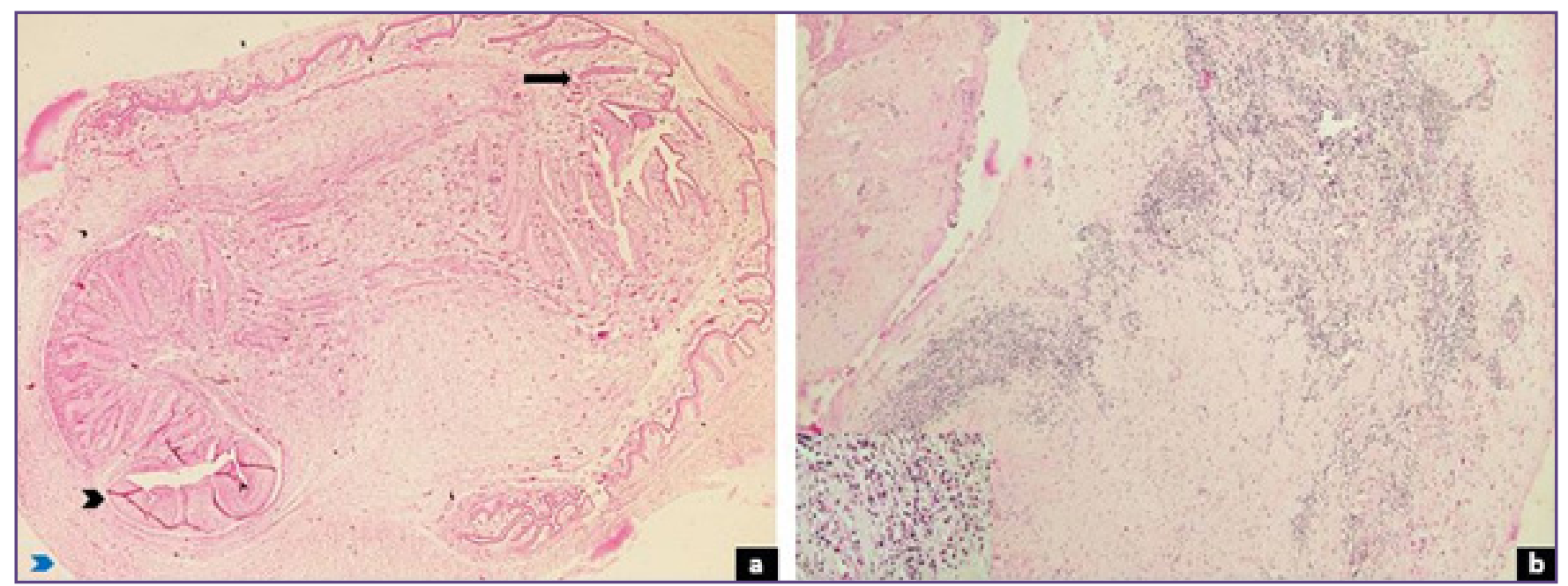

Fig. 3(a-b): (a) Larval form of T. solium with a fibrous pseudocapsule (blue arrowhead)with duct-like invaginations (arrow) along with one pair of sucker (black arrowhead), H\&E, 40X; (b) inflammatory infiltrate in surrounding tissue comprising of lymphocytes and plasma cells, H\&E, 40X (inset-400X).

${ }^{[8]}$. Even though intracranial neurocysticercosis is a well known parasitic infestation of CNS worldwide, intraspinal involvement, and particularly intramedullary NCC, is extremely rare even in endemic areas despite being known to coexist with intracranial NCC. ${ }^{[1,4]}$ George A Alsina et al (2002) in their study brought out less than 200 reported cases of spinal NCC worldwide even after including three fourth cases having an underlying/associated intracranial NCC. ${ }^{[9]}$ It is postulated that approximately 100 fold blood supply of brain parenchyma as compared to spine is responsible for an increased incidence of intracranial NCC and intramedullary lesion results from direct hematogenous or ventriculo-ependymal spread. ${ }^{[10]}$

Intramedullary NCC occurs most often in young adults between 20-45 years of age. ${ }^{[2]}$ Most common clinical signs and symptoms include paraparesis, myelopathy, radiculopathy, spasticity and bowel and bladder incontinence. ${ }^{[1,2]}$ In the presence of known history of intracranial NCC, as in our case, one has to suspect possibility of intramedullary NCC besides keeping other causes of paraparesis in mind. Neurological examination of the patient may help to localize the site of neurological deficits. MRI with contrast enhancement is the gold standard for diagnosing intramedullary NCC as it provides high quality resolution with multi sequential and multiplanar imaging. Also, MRI helps the treating physicians/ surgeons to decide the treatment plan between drug therapy or surgery or drug therapy followed by surgery based on the pathological stage of NCC and severity of compression. ${ }^{[10]}$ Cerebrospinal fluid (CSF) examination can be informative; however, it is rarely done as it may exaggerate neurological symptoms. CSF reveals lymphocytic and/or eosinophilic pleocytosis with normal glucose levels and elevated protein levels. ${ }^{[4]}$ Persistent blood eosinophilia may hint towards an active disease though it is not a reliable finding. Haematological investigations were normal in our case and CSF was not done due to the above-mentioned risks. Characteristic MRI findings followed by histopathology of the excised mass helped in confirming the diagnosis besides ruling out tumors and other non-tumorous conditions of spine.

Treatment for intramedullary NCC is either drug therapy with albendazole or surgery. In cases where intramedullary NCC leads to cord or nerve root compression, laminectomy and excision of the lesion is preferred to help decompression of the nerve roots as well as for establishing histopathological diagnosis on the excised tissue. Steroids are used preoperatively to reduce the inflammatory response. ${ }^{[7]}$ Histopathologically, there are four stages of NCC; (a) vesicular stage: viable larval form of organism is seen inside a vesicle (cystic cavity) which comprises of an invaginated scolex surrounded by eosinophilic double layered membrane. One larva has two pairs of suckers and hooklets to attach with the cystic cavity; ${ }^{[11]}$ (b) colloidal vesicular stage: larval degeneration begins and induces lymphoplasmacytic inflammatory response, fibrosis and necrosis in the surrounding tissue; ${ }^{[11]}$ (c) granular-nodular stage: there is progressive degeneration of the larval stage with beginning of calcification of the larva and involution of cystic cavity; ${ }^{[11]}(d)$ nodular-calcified stage: here, larva is replaced by collagen and calcification. ${ }^{[11]}$ Our case had the viable larval form inside a vesicle. 


\section{Conclusion}

Though endemic in low socio-economic countries like India, the incidence of spinal and particularly, intramedullary NCC is very rare. Laminectomy is preferred in intramedullary NCC so as to relieve the compression symptoms. Histopathological examination of the excised mass in correlation with clinical and radiological inputs can help in establishing a correct diagnosis.

\section{Abbreviations and Symbols \\ CSF: Cerebrospinal fluid}

CE MRI: Contrast Enhanced Magnetic Resonance Imaging

DV: Dorsal Vertebra

MRC: Medical Research Council

NCC: Neurocysticercosis

\section{Ackwoledgements}

Nil

\section{Funding}

Nil

\section{Competing Interests}

There are no competing interests.

\section{Statement of Informed Consent}

The authors certify that they have obtained all appropriate patient consent forms. In the form the patient(s) has/have given his/her/their consent for his/her/their images and other clinical information to be reported in the journal. The patient(s) understand that their names and initials will not be published and due efforts will be made to conceal his/ her/their identity, but anonymity cannot be guaranteed.

\section{References}

1. Maste PS, Lokanath YK, Mahantshetti SS, Soumya S. Isolated Intramedullary Spinal Cysticercosis : A Case Report with Review of Literature of a Rare Presentation. Asian J Neurosurg 2018;13:154-6.

2. Qi B, Ge P, Yang H, Bi C, Li Y. Spinal Intramedullary Cysticercosis : A Case Report and Literature Review. Int J Med Sci 2011;8:420-3.

3. Kim M, Rhim SC, Khang SK. Intramedullary Spinal Cysticercosis : A Case Report and Review of Literature. Korean J Spine 2014;11:81-4.

4. Qazi Z, Ojha BK, Chandra A, Singh SK, Srivastava C, Patil TB. Isolated Intramedullary Spinal Cord Cysticercosis. J Neurosci Rural Pract 2014;5:S66-8.

5. Noguera EMS, Sic RP, Solis FE. Intramedullary Spinal Cord Neurocysticercosis Presenting as Brown-Séquard syndrome. BMC Neurol 2015;15:1-4.

6. Coyle CM, Tanowitz HB. Diagnosis and Treatment of Neurocysticercosis. Interdiscip Perspect Infect Dis 2009;2009:180742.

7. Muralidharan V, Nair BR, Patel B, Rajshekhar V. Primary Intradural Extramedullary Cervical Spinal Cysticercosis. World Neurosurg [Internet] 2017 Oct;106:[about 6p.]. Available from: http://dx.doi.org/10.1016/j. wneu.2017.07.008

8. Brutto OHD. Neurocysticercosis : A Review. ScientificWorldJournal 2012;2012:159821.

9. Alsina GA, Johnson JP, Mc Bride D, Rhoten PRL, Mehringer $\mathrm{CM}$, Stokes JK. Spinal neurocysticercosis. Neurosurg Focus 2002;12:1-7.

10. Mathuriya SN, Khosla VK, Vasishta RK, Tewari MK, Pathak A, Prabhakar S. Intramedullary Cysticercosis : MRI Diagnosis. Neurol India 2001;49:71-4.

11. Moskowitz J, Mendelsohn G. Neurocysticercosis. Arch Pathol Lab Med 2010;134:1560-3.

*Corresponding author:

Dr. Puneet Baveja, Armed Forces Medical College, Pune, Maharashtra, India

Phone: +91 7042166657

Email: pbaveja@gmail.com,

Date of Submission : 03/08/2020

Date of Acceptance : 27/09/2020

Financial or other Competing Interests: None.
Date of Publication : 30/11/2020 